\title{
Relación entre los gastos asociados a la Ley de Subvención Escolar Preferencial (SEP) y resultados Simce de colegios básicos subvencionados en 2014*
}

\author{
Leonardo Andrés Villagrán Chicago** \\ Juan Pablo Dreyse Muñoz ${ }^{\star \star *}$
}

\begin{abstract}
RESUMEN
Alcanzar una educación de calidad y equitativa, como es declarado en nuestra legislación, es necesario para que todos los estudiantes tengan acceso y se les permita obtener distintos tipos de logros en sus aprendizajes, lo que resulta más complejo en establecimientos que presenten altos índices de vulnerabilidad. Es por esta razón que el estado de Chile entrega recursos extra a partir de la Subvención Escolar Preferencial (Ley SEP 20.248), para la creación de Planes de Mejoramiento Educativo (PME), y la Agencia de la Calidad de la Educación realiza evaluaciones para medir el rendimiento de los estudiantes a través de las pruebas SIMCE, que en base a sus resultados permite año a año generar sugerencias y remediales a los procesos de enseñanza y aprendizaje, en función de la calidad de la educación. La presente investigación, que se enmarca en un paradigma cuantitativo, buscó determinar la relación entre los recursos entregados por la Subvención Escolar Preferencial y los resultados promedio de evaluaciones SIMCE de establecimientos subvencionados en 20I4. De esta forma dar un paso inicial en la búsqueda del uso eficiente de recursos públicos, para el mejoramiento de los resultados de aprendizajes de los estudiantes de nuestro país.
\end{abstract}

Palabras clave: SEP, SIMCE, gestión de recursos.

Relationship between expenses associated with the Preferential School Grant (SEP) Law and SIMCE results in subsidized primary schools in 2014

\section{ABSTRACT}

Achieving a quality and equitable education, as stated in our legislation, is necessary for all students have access and are able to obtain expected levels of achievement in learning, which is more complex in schools with high levels of vulnerability. For this reason, the State of

* Este artículo procede de: Villagrán, L. (2017). Relación entre los gastos asociados a la ley de subvención escolar preferencial de cada región de Chile y los resultados de las evaluaciones SIMCE de las asignaturas de lenguaje y matemática de colegios básicos municipales y particular subvencionados en el año 2014 (tesis de magíster). Chile: Universidad Católica Silva Henríquez.

* Magíster en Educación Mención en Gestión y Liderazgo Pedagógico de la Universidad Católica Silva Henríquez, Chile-. E-mail: leonardovillagran@yahoo.com

Magíster en Educación Mención Gestión Educativa y Docente Universidad Católica Silva Henríquez, Chile-. E-mail: jdreysem@ucsh.cl 
Chile provides extra resources by a Preferential School Grant (Law SEP 20,480), for the creation of Educational Improvement Plans (PME). The Agency for Quality Education carries out assessments to measure students' performance through the SIMCE tests which, based on their results, year after year allow generating suggestions and improvements to teaching and learning processes, in function of the quality of education. The present research, which is part of a quantitative paradigm, was aimed to determining the relationship between resources provided by the Preferential School Grant and the average results of SIMCE evaluations of subsidized institutions in the year 20I4. In this way, it was possible to take an initial step in searching for the efficient use of public resources, the improvement of learning results by students of our country.

Keywords: SEP, SIMCE, resource management.

\section{Introducción}

La educación ha jugado un papel fundamental en el desarrollo y progreso de las distintas comunidades del mundo, porque permite al ser humano volverse parte de la sociedad y en ella descubrir el lugar que ocupará al seleccionar su profesión (Pastrana, Fernández, Salinas, Gutierrez \& Nuñez, 2015). Sin embargo, dicha tarea no es simple y menos para estudiantes que se encuentran en situación de vulnerabilidad, los cuales poseen desventajas en los procesos de enseñanza, carencia de recursos pedagógicos adecuados, falta de acceso a la educación y entornos que no producen impactos en los aprendizajes (Weinstein, Fuenzalida \& Muñoz, 2010).

Por esta razón, los países han encausado sus políticas para desarrollar programas de mejoramiento educativo, centrados en la calidad y equidad para todos sus estudiantes.

En Chile, además de la subvención tradicional o regular que se dispone para la educación pública, otra manera en que el Estado entrega recursos extra es a través de la Subvención Escolar Preferencial (Ley 20.480), también conocida como ley SEP, para la creación de acciones que permitan a los establecimientos generar un aumento en los niveles de logro, disminuyendo la brecha de los aprendizajes entre los estudiantes de distintos grupos sociales. Los resultados de la implementación de este programa se miden a partir de los resultados del sistema de evaluación nacional SIMCE, por lo cual es prioridad incrementar el desempeño de los resultados 
de estas evaluaciones, ya que será el factor que dará cuenta del mejoramiento de los aprendizajes de estudiantes (Román \& Murillo, 20I2).

Por lo tanto, la presente investigación, que da base al artículo, tuvo por objeto determinar la relación entre los recursos financieros SEP entregados a establecimientos educacionales a partir de la Ley de Subvención Escolar Preferencial de cada región del país y los resultados promedio de evaluaciones de SIMCE de las asignaturas de lenguaje y matemática de los cursos cuarto y octavo básico en 20I4. De esa forma se estableció si existe una asociación entre los parámetros analizados y, por otra parte, se obtuvo la magnitud de la relación encontrada.

Para llevar a cabo esta investigación fue necesario utilizar datos de organismos vinculados al Sistema Nacional de Aseguramiento de la Calidad Educativa, sobre montos de gastos y categorías asociadas a los recursos financieros SEP de establecimientos subvencionados, los cuales fueron entregados por la Superintendencia de Educación; por otra parte, los resultados promedios de evaluaciones SIMCE fueron obtenidos a través de la Agencia de la Calidad de Educación, para realizar un análisis de la información más relevante que permitiera conocer y responder a las preguntas planteadas.

\section{Justificación de la investigación}

Dado que los planes de mejoramiento educativo suscritos a la ley SEP deben influir en el rendimientos de los estudiantes de establecimientos que reciban este tipo de subvención, es necesario determinar que existe una relación significativa entre los recursos adicionales entregados por el Estado y el mejoramiento de los aprendizajes de los estudiantes, donde solo se busca establecer una asociación entre variables.

La idea fue enfocar este estudio como un punto de partida para futuras investigaciones, las cuales podrían determinar si se está realizando un uso adecuado y pertinente de los recursos SEP o, por el contrario, se está generando un impacto negativo a nivel país o simplemente no existe ninguna relación con los niveles de logro 
que se desea alcanzar, lo que podría representar la necesidad de una reestructuración de las políticas públicas de subvención en este ámbito de la educación.

Para identificar el problema de investigación es necesario definir las variables que determinarán el estado de cada establecimiento analizado (nivel de logro en los procesos de mejoramiento educativo). Por esta razón se ha seleccionado los resultados de las evaluaciones del sistema de evaluación nacional (SIMCE) como un medio para determinar "el desempeño de los estudiantes en diferentes sectores de aprendizaje del currículum nacional, y relacionándolo con el contexto escolar y social en el que estos aprenden" (MINEDUC, 20I2c, p. 4), para compararlos con los gastos realizados a partir de los recursos financieros SEP que recibe cada establecimiento subvencionado adscrito al programa. Estos montos de subvención son entregados para el mejoramiento de la calidad escolar, ya que permiten "compensar las desigualdades sociales de origen que afectan a los niños y niñas de los sectores más modestos, entregando un aporte adicional a los establecimientos que los acogen y comprometiendo con ellos una estrategia de mejoramiento educativo" (Weinstein, Fuenzalida \& Muñoz, 2010, p. I62).

Por lo cual es de suma importancia determinar si existe una relación que dé cuenta del impacto en los resultados académicos en lenguaje y matemática, ya que respecto del plan de mejoramiento educativo contenido en la SEP, "su fin último es mejorar los aprendizajes de los estudiantes (especialmente de los prioritarios y de menor rendimiento), e incrementar los desempeños del SIMCE (de manera prioritaria en Lenguaje y Matemáticas)" (Román \& Murillo, 2012, p. 57). Por lo tanto la pregunta de investigación se presentó de la siguiente forma: ¿Existe una relación significativa entre los gastos de recursos financieros SEP utilizados por establecimientos de tipo municipal y particular subvencionado de las distintas regiones de Chile y sus resultados de puntajes SIMCE de las asignaturas de lenguaje y matemática de los cursos cuarto y octavo básico en el año 20I4? 


\section{Objetivo general}

Para responder al problema definido y determinar la relación entre los gastos de recursos financieros SEP de establecimientos de tipo municipal y particular subvencionado de cada región de Chile y los resultados de puntajes SIMCE de las asignaturas de lenguaje y matemática de los cursos de cuarto y octavo básico en el año 20I4, se determinó el siguiente objetivo general: establecer la relación entre los gastos de recursos financieros SEP utilizados por establecimientos de tipo municipal y particular subvencionado de cada región de Chile y los resultados de puntajes SIMCE de las asignaturas de lenguaje y matemática de los cursos de cuarto y octavo básico en el año 2014.

\section{El sistema educativo en Chile y su financiamiento}

El sistema escolar chileno se caracteriza por establecer un tipo de financiamiento de la educación que integra tanto al sector público como al sector privado, con el fin de salvaguardar los derechos de libertad de enseñanza y acceso a la educación establecidos en la Constitución. Con base en la proveniencia de los recursos, actualmente podemos referirnos a tres tipos de colegios: particulares pagados, municipales y particulares subvencionados.

Los colegios del tipo particular pagado, quienes concentran el 7,6\% de las matrículas para el año 2015 (Centro de Estudios MINEDUC, 20I5) se caracterizan por ser de propiedad privada y por no contar con fondos públicos, ya que su financiamiento se genera a través de aportes de privados y del aporte de la misma comunidad educativa, recayendo la responsabilidad monetaria principalmente en las familias a través del pago de matrícula y arancel; "son aquellos que más fielmente representan una educación propiamente privada, puesto que se financian sólo con ingresos propios ya sea de las matrículas que cobran a las familias de los alumnos o bien a partir de otros ingresos privados" (Corvalán, Elacqua \& Salazar, 2009, p. 8).

Por otro lado, los colegios municipales son aquellos establecimientos bajo administración municipal, cuyo financiamiento deriva principalmente de recursos públicos obtenidos a través de la 
subvención a la demanda, garantizando una opción gratuita de acceso a la educación para las familias chilenas. Mediando entre las características de la educación pública y la educación privada se encuentran los colegios del tipo particular subvencionado. Podemos definir a este tipo de colegios como "aquellos establecimientos que, siendo de propiedad privada, reciben la totalidad o parcialidad de sus recursos de fondos estatales (Corvalán, Elacqua \& Salazar, 2009, p. 4)”. Al igual que en los establecimientos municipales, los colegios del tipo particular subvencionado reciben financiamiento público a través de la subvención a la demanda educativa, asignándosele una cierta cantidad de recursos por cada alumno que asiste al establecimiento; sin embargo, al igual que en la educación privada, este tipo de establecimientos pueden establecer un copago a las familias a través del cobro de matrícula y arancel.

De esta forma, los establecimientos particulares subvencionados se surten tanto de recursos públicos como privados; sin embargo, el distinto grado de prevalencia de los distintos tipos de recursos genera que exista una gran diversidad en este tipo de establecimientos en relación con la cantidad de recursos a su disposición.

\section{Importancia de las asignaturas de lenguaje y matemática}

El Ministerio de Educación considera el desarrollo del lenguaje como uno de sus objetivos fundamentales, ya que es la principal herramienta mediante la cual los seres humanos pueden aprehender el mundo a través de la comunicación interpersonal e intrapersonal. "El lenguaje es la forma que toma nuestro pensamiento, nos relaciona con los demás y nos hace parte de una comunidad cultural" (MINEDUC, 2012a, p. 34).

La importancia de esta asignatura en la sociedad chilena radica en que es en el proceso educacional donde los estudiantes pueden adquirir y potenciar las habilidades comunicativas necesarias para enfrentar las problemáticas de la vida cotidiana, adquirir nuevos conocimientos y lograr una mayor comprensión de la realidad, siendo fundamental para el desarrollo del pensamiento crítico. Por otro lado, el Ministerio de 
Educación señala que el desarrollo del lenguaje permite generar un sentido de identidad y pertenencia en los alumnos mediante la lengua.

Al igual que el lenguaje, la matemática es parte del desarrollo de la humanidad, convirtiéndose en una herramienta fundamental para los estudiantes al momento de integrarse en la sociedad y enfrentar problemáticas cotidianas. "La matemática es en sí misma un aspecto importante de la cultura humana: es una disciplina cuya construcción empírica e inductiva surge de la necesidad y el deseo de responder y resolver situaciones provenientes de los más variados ámbitos” (MINEDUC, 2012b, p. 87). La importancia de esta asignatura radica en que los conocimientos matemáticos permiten una mayor comprensión del mundo mediante la resolución de problemas y el desarrollo del pensamiento crítico. El Ministerio de Educación señala que el aprendizaje de esta materia implica el desarrollo de habilidades cognitivas, como la visualización, la representación, la modelación, la simulación y la conjeturación, contribuyendo al desarrollo del pensamiento lógico. Además, las matemáticas se presentan como un lenguaje universal, siendo una herramienta de comunicación presente en la mayoría de las disciplinas.

\section{Evaluaciones SIMCE}

En 2012, la Agencia de la Calidad de la Educación se hizo cargo de este sistema de evaluación, y se ha encargado, desde esa fecha, de realizar análisis de los resultados de aprendizajes obtenidos para medir los logros alcanzados en los contenidos y habilidades vigentes dentro del currículo educativo, enfocándose en distintas áreas, mediante la realización de evaluaciones a estudiantes en niveles de enseñanza específicos y la aplicación de encuestas a la comunidad educativa, las cuales permiten entregar un contexto adecuado para el análisis de los resultados obtenidos dentro de las diferentes realidades que se pueden encontrar en el país.

Estos resultados permiten, a los establecimientos educacionales, obtener información de los logros alcanzados por sus estudiantes en comparación con el nivel nacional y de esta forma determinar cuáles son sus fortalezas y debilidades, permitiendo, mediante la 
retroalimentación, desarrollar nuevas estrategias que contribuyan al mejoramiento de los aprendizajes (MINEDUC, 2016).

\section{Ley de Subvención Escolar Preferencial SEP}

Los aportes de recursos extra para la educación corresponden a la Ley N 20.248 de la Subvención Escolar Preferencial (2008), conocida también como ley SEP, su función corresponde al mejoramiento de la calidad y equidad de educación de establecimientos subvencionados, entregando recursos financieros que deben estar asociados un aumento de los aprendizajes de los estudiantes; es por esto que se deben generar compromisos con toda la comunidad educativa que participa en los procesos de enseñanza.

\section{Rendición de recursos financieros SEP}

Con el acceso a recursos financieros extra, a través de los ingresos entregados por el Ministerio de Educación, cada establecimiento subvencionado que esté adherido a la ley SEP debe realizar una rendición de gastos anual, los cuales deben haber sido utilizados para cumplir exclusivamente las acciones relacionadas con el plan de mejoramiento educativo (PME) dentro de las cuatro áreas comprendidas por este (gestión curricular, liderazgo, convivencia y gestión de recursos), las cuales permitan optimizar los resultados de aprendizajes de los estudiantes y con un buen uso "-en el corto y mediano plazo-, pueden contribuir sustantivamente al mejoramiento de los procesos y resultados educacionales" (MINEDUC, 20I4, p. 5).

La rendición de los gastos SEP utilizados por cada establecimiento es una obligación legal que cada sostenedor debe efectuar a la Superintendencia de Educación, en donde:

El Decreto Supremo No 469 del 20I4, en su Art. No 2, letra a), define la rendición de cuenta pública del uso de los recursos como "la obligación legal de los sostenedores de establecimientos educacionales subvencionados o que reciban aportes del Estado, de dar a conocer y entregar las cuentas 
comprobadas del uso de todos los recursos públicos y privados que administren o perciban, anualmente, en la forma y plazo que establece el presente Reglamento, de acuerdo a los instrumentos y formatos estandarizados que fije la Superintendencia de Educación, y bajo su fiscalización”. (Superintendencia de Educación, 2016, p. 5)

\section{Enfoque}

La investigación se enmarcó en un paradigma cuantitativo de tipo transeccional correlacional. Esto se debe fundamentalmente al problema presentado, el cual corresponde a determinar la relación que existe entre los gastos asociados a la ley de subvención escolar preferencial de cada región de Chile y los resultados de las evaluaciones SIMCE de las asignaturas de lenguaje y matemática de colegios básicos municipales y particulares subvencionados en 20I4. En esta investigación se buscó encontrar el tipo de relación y correlación entre variables, las cuales no varían al ser medidas en un periodo de tiempo definido y no son alteradas durante el estudio. Además, se realizaron pruebas de hipótesis, las cuales fueron aceptadas o rechazadas para determinar si existía una relación estadísticamente significativa y la magnitud de la correlación encontrada.

\section{Población y muestra}

La población para esta investigación $(\mathrm{N})$ corresponde a las instituciones de educación que sean parte del programa de Subvención Escolar Preferencial SEP de cada región del país, que estén dentro de los registros de la Superintendencia de Educación y validados en 20I4. Además, la dependencia de los establecimientos municipales debe ser de tipo corporación municipal o DAEM, y para el caso de los colegios privados que reciben aportes del Estado su dependencia debe ser de tipo particular subvencionado. El total de la población para este estudio está constituido por 7.990 establecimientos de todo el país, de los cuales 5.068 corresponden a colegios pertenecientes a dependencia municipal y 2.922 a colegios de dependencia particular subvencionada. 
Las muestras (n) corresponden a las instituciones de educación que sean parte del programa de subvención escolar preferencial SEP, que hayan utilizado los recursos financieros asignados para realizar gastos específicos dentro de las categorías de gastos en remuneraciones, gastos en asesoría técnica y capacitación, gastos en recursos de aprendizaje, gastos en equipamiento de apoyo pedagógico y gastos de operación, y que tengan registros de evaluaciones SIMCE de lenguaje y/o matemática para el periodo comprendido por el año 2014 para los cursos cuarto y/u octavo básico de cada región de Chile.

Es importante destacar que la construcción de las muestras se basó en los registros de instituciones que cumplieron los requisitos necesarios para llevar a cabo pruebas de correlación, las cuales se indican a continuación:

- Las instituciones debían poseer registros de resultados promedio de evaluaciones SIMCE en el periodo 20I4, para las asignaturas de lenguaje y/o matemática de los cursos cuarto y/u octavo básico. Además los resultados deben fueron validados por la Agencia de la Calidad de la Educación.

\section{Operacionalización de las variables}

Como primera dimensión se asignó el nombre "recursos financieros SEP” a los gastos más representativos de montos de subvención escolar preferencial que han sido utilizados por cada institución analizada (ítems que consideran mayor gasto a nivel nacional), éstas corresponden a variables cuantitativas, dado que se evaluará la capacidad que tiene cada una de ellas de relacionarse con otras variables, y serán de tipo continua, ya que pertenecen a valores expresados en moneda nacional.

La segunda dimensión fue llamada "resultados SIMCE”, dado que agrupa variables con los puntajes promedio de evaluaciones de lenguaje y matemática de las mismas instituciones referidas en la dimensión anterior; éstas corresponden a variables cuantitativas, ya que son de tipo continuas, presentadas como puntajes promedios de evaluación de las asignaturas de lenguaje y matemática para los cursos cuarto y octavo básico. 
En cuanto a la dimensión resultados SIMCE, se ha seleccionado las asignaturas de lenguaje y matemática en cuarto y octavo básico, dado que son las evaluaciones que poseen mayor número de aplicaciones desde su creación y por su importancia dentro del currículo nacional, ya que desde 2004 se ha puesto énfasis en el desarrollo de programas y planes relacionadas con estas dos asignaturas como factores determinantes para el mejoramiento educativo de la enseñanza básica y de esta forma influir en la calidad, equidad y retención de los estudiantes del sistema subvencionado (Marshall Infante, 2004).

\section{Fundamentación, descripción de instrumentos y técnicas}

Los datos tabulados, que comprenden la dimensión recursos financieros SEP, fueron obtenidos a través de un requerimiento a la Superintendencia de Educación; por otro lado, los datos que corresponden a la dimensión resultados SIMCE fueron solicitados y entregados por la Agencia de la Calidad de la Educación, para ambos casos los datos recolectados corresponden al año 20I4. La tabulación final se llevó a cabo conjugando las variables de las dos dimensiones en una sola base de datos, a partir del RBD de cada institución y separando las instituciones de cada región por el tipo de institución, ya sea municipal o particular subvencionado.

\section{Análisis correlacional:}

Para obtener la relación entre las variables de las dimensiones recursos financieros SEP y resultados SIMCE se realizaron pruebas de hipótesis a través de la correlación de Pearson y de esta forma obtener la relación que existe en cada región del país y, al mismo tiempo, responder la pregunta de investigación que está enunciada dentro del objetivo general.

Las pruebas se realizaron para todos los establecimientos de tipo municipal y particular subvencionado que cumplían la condición de tener gastos dentro de la dimensión recursos financieros SEP y puntajes de evaluaciones dentro de la dimensión resultados SIMCE, para el periodo comprendido por el año 2014. 
Es muy importante destacar que en esta investigación se buscó obtener la relación y no causalidad, es decir, solo se quiere determinar la asociación que existe y no demostrar cómo influyen las variables de la dimensión recursos financieros SEP en las variables de la dimensión SIMCE, ya que "Correlación y causalidad son conceptos asociados, pero distintos. Si dos variables están correlacionadas, ello no necesariamente implica que una será causa de la otra" (Hernández, Fernández \& Baptista, 2010, p. 100).

\section{Resultados del análisis correlacional asociados al objetivo general}

A partir de las pruebas de correlación realizadas a las distintas categorías de gastos de recursos financieros SEP y los resultados promedio de evaluaciones SIMCE, se determinó que existe una relación significativa entre los gastos de recursos financieros SEP utilizados por establecimientos subvencionados de las regiones de Chile y los resultados de puntajes SIMCE de las asignaturas de lenguaje y matemática de los cursos de cuarto y octavo básico en el año 2014, con la excepción de la primera y tercera regiones. Es importante destacar que al realizar el mismo análisis por el tipo dependencia de los establecimientos en estudio, se obtuvo que:

- Existe una relación significativa entre los gastos de recursos financieros SEP utilizados por establecimientos de tipo municipal y los resultados de puntajes SIMCE de las asignaturas de lenguaje y matemática de los cursos de cuarto y octavo básico en el año 20I4, con la excepción de la primera, tercera, cuarta, quinta, sexta, decimosegunda y decimoquinta regiones del país.

- Existe una relación significativa entre los gastos de recursos financieros SEP utilizados por establecimientos de tipo particular subvencionado y los resultados de puntajes SIMCE de las asignaturas de lenguaje y matemática de los cursos de cuarto y octavo básico en el año 20I4, con la excepción de la primera, tercera, y decimoprimera regiones del país. 


\section{Hallazgos obtenidos por región}

El objetivo principal del estudio fue responder a la pregunta de investigación, en la cual se pudo concluir que existe una relación entre los gastos de recursos financieros SEP utilizados por establecimientos de tipo municipal y particular subvencionado de las regiones de Chile y sus resultados de puntajes SIMCE de las asignaturas de lenguaje y matemática de los cursos cuarto y octavo básico en 20I4, con la excepción de la primera y tercera regiones. Sin embargo, la realidad del estudio determinó que de las 600 pruebas de correlación realizadas, solo se obtuvo I34 relaciones significativas, lo que equivale a 22,3\% del total; dicho de otra forma, se obtuvo 466 resultados, en los que no se encontró relaciones significativas, lo que equivale al $77,7 \%$ del total.

Gráfico $\mathrm{N}^{\circ} \mathrm{I}$. Porcentaje de resultados de relaciones entre gastos de recursos financieros SEP y resultados de evaluaciones SIMCE de establecimientos subvencionados.

\section{PORCENTAJE DE RESULTADOS DE RELACIONES ENTRE VARIABLES}

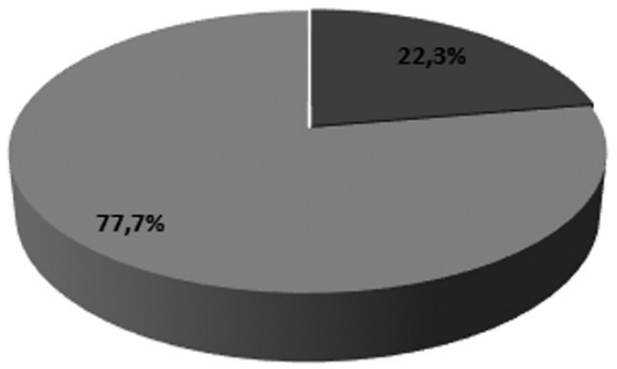

- RELACIONES SIGNIFICATIVAS

- SIN RELACIONES SIGNIFICATINAS

Dado que los recursos financieros SEP fueron destinados para el mejoramiento de los niveles de aprendizaje, un aumento de estos montos solo se relaciona con el aumento de los resultados de los puntajes SIMCE en un poco más del 20\%, lo que puede parecer bajo e inquietante, pero al mismo tiempo se podría transformar en una oportunidad para realizar nuevos estudios en materia de subvención SEP. 
Al dirigir la atención a los casos donde existen relaciones significativas, 92 de los casos corresponden a relaciones con una magnitud de correlación positiva baja, lo que equivale a un $68,7 \%$ del total, lo que marca una tendencia en los resultados; lo sigue la magnitud positiva moderada, con 23 casos, que equivale a un $17,2 \%$ del total; en forma posterior las magnitudes negativa fuerte, negativa débil y positiva fuerte con 5 casos cada una, que representa 3,7\% del total, y por último las magnitudes negativa moderada y ninguna o muy débil, con 2 casos que equivalen a 1,5\% del total.

Respecto de la conclusión anterior, es importante considerar que existen relaciones positivas, no obstante la mayoría son bajas, lo que podría suponer que el uso de recursos financieros SEP no está orientado como debería para el mejoramiento de los aprendizajes de los estudiantes de establecimientos subvencionados, que son evaluados a través de las pruebas SIMCE a nivel nacional. Además, existen casos de alerta, como la segunda región, la cual posee 8 relaciones de magnitud negativa, de las cuales 4 son fuertes, I moderada y 3 débiles, lo que podría significar la necesidad de realizar estudios de corte cualitativo que se enfoquen en la particularidad de la zona, para evaluar a fondo lo ocurrido en dicha región. También se podría evaluar los casos de la decimoprimera y decimoquinta región, las cuales son las únicas que registran una magnitud positiva fuerte, ya que ello podría entregar información valiosa respecto de lo que produce dicho efecto y cómo reproducirlo en otras localidades del país.

Al observar los resultados, pero esta vez considerando el tipo de dependencia de las instituciones de educación, se obtuvo que de las 134 relaciones significativas encontradas, IOI pertenecen a colegios particulares subvencionados y 33 a colegios de tipo municipal; si se consideran las 600 pruebas realizadas, I6,8\% de los casos encontrados corresponden a instituciones de tipo particular subvencionado y solo 5,5\% corresponde a colegios municipalizados. Los datos obtenidos vuelven a centrar su atención en los colegios particulares con subvención del Estado, en lo que, además de tener mejores resultados en las evaluaciones SIMCE también los tienen en las relaciones encontradas, ya que superan por más de II puntos porcentuales a la educación municipal. 


\section{Conclusiones destacadas a partir de resultados por categoría de gasto SEP y dependencia de loS establecimientos de educación}

Dado que la investigación corresponde a un enfoque cuantitativo, el estudio se concentró en obtener resultados que entreguen una generalización de los datos, lo cual no atiende la particularidad de cada región, comuna o zona del país; sin embargo, ciertos resultados destacan por sobre otros a partir de las características de correlación y magnitud encontradas. Por esta razón, se describirán puntos críticos de resultados de la relación que existe entre los gastos de recursos financieros SEP y los resultados de puntajes de evaluaciones SIMCE en 2014.

- En la Segunda Región existen 8 relaciones significativas con correlación negativa, es decir, a medida que suben los montos de los gastos de recursos financieros SEP, los resultados de puntajes de evaluaciones SIMCE bajan. La relación obtenida es lo contrario a lo que se busca; por esta razón se considera como punto de interés, ya que se podría analizar qué factores intervienen, para cambiar dicha condición, donde las categorías involucradas son gastos en remuneraciones y gastos en equipamiento de apoyo pedagógico, gastos en recursos de aprendizaje y gastos de operaciones.

- En la Región Metropolitana, en dependencias de tipo particular subvencionado, se puede encontrar varias relaciones significativas con correlación positiva entre montos de gastos de recursos financieros SEP y los resultados de puntajes de evaluaciones SIMCE; sin embargo, no existen relaciones significativas para casos que contengan gastos de asesoría técnica y capacitación. En dependencias de tipo municipal, todos los resultados que consideran categorías de gastos de asesoría técnica y capacitación poseen una relación significativa, no obstante, muy pocos resultados consideran las otras categorías de gasto. Esto se podría interpretar como un uso ineficiente de los recursos de asesoría y capacitación por parte de los establecimientos de dependencia particular subvencionada y un buen uso de los otros gastos bajo 
estudio. Por el contrario, se podría interpretar que hay un buen uso de los recursos de asesoría y capacitación por parte de los establecimientos de dependencia municipal y un uso ineficiente de los otros gastos bajo estudio.

- En la Decimoprimera Región existen 2 relaciones significativas con correlación positiva fuerte para colegios municipalizados, es decir, a medida que suben los montos de los gastos de recursos financieros SEP, los resultados de puntajes de evaluaciones SIMCE también aumentan. La relación obtenida es lo que se busca, por esta razón se considera como punto de interés, ya que se podría analizar qué factores intervienen para replicar los resultados en otros establecimientos que no posean dicha condición, tanto para dependencias de tipo municipal como particular subvencionado.

- En la Decimoquinta Región existen 3 relaciones significativas con correlación positiva fuerte para colegios de tipo particular subvencionado, es decir, a medida que suben los montos de los gastos de recursos financieros SEP, los resultados de puntajes de evaluaciones SIMCE también aumentan. La relación obtenida es lo que se busca, por esta razón se considera como punto de interés, ya que se podría analizar qué factores intervienen para replicar los resultados en otros establecimientos que no posean dicha condición, tanto para dependencias de tipo municipal como particular subvencionado.

\section{Conclusión}

Dada la investigación y su enfoque cuantitativo se pudo determinar una baja relación entre recursos financieros SEP y resultados de evaluaciones SIMCE. Es por esto que es necesario enfatizar la realización de nuevos estudios, los cuales centren su atención en el impacto producido por las políticas públicas en el uso de recursos SEP, colocando mayor énfasis en los establecimientos de dependencia municipal, pero sin dejar de lado a la educación particular subvencionada, y de esta forma realizar diagnósticos que permitan implementar Planes de Mejoramiento Educativo (PME) que apunten de forma eficiente y eficaz a perfeccionar los niveles 
de logro de los aprendizajes de los estudiantes y al mismo tiempo desarrollar un sistema que responda a la necesidad de una educación equitativa y de calidad para todos. Por otra parte, es importante destacar que el sistema educativo requiere de ajustes, monitoreo y precisiones que mejoren la distribución de los recursos públicos, que considere las diferencias de cada región y que ponga acento en la descentralización, y de una gestión por parte del Estado que dé cuenta de los verdaderos desafíos de la educación, en cuanto al uso de los recursos financieros y al mejoramiento de los resultados que cada niño de este país necesita para crecer, sin importar la realidad de la cual provenga. Además, que permita un desarrollo integral, donde el alumno adquiera las herramientas necesarias para enfrentar los desafíos de su futuro laboral y, al mismo tiempo, ser un gran aporte para la creación de una nueva comunidad escolar más justa e inclusiva. El desafío es hacernos cargo de cómo se utilizan los recursos, comprendiendo que no solo es un tema de aumentar anualmente el presupuesto en educación, sino de ver como se distribuye con una gestión oportuna y eficiente.

\section{Referencias bibliográficas}

Centro de Estudios MINEDUC. (2015). Variación de matrícula y tasas de permanencia por sector. Recuperado de http:/ / centroestudios.mineduc.cl/ tp_enlaces/portales/tp5996f8b7cm96/uploadImg/File/Evidencias/ Evidencias\%20final_julio_20I5.pdf

Corvalán, J., Elacqua, G. \& Salazar, F. (2009). El sector particular subvencionado en Chile. Tipologización y perspectivas frente a las nuevas regulaciones. Santiago, Chile: Ministerio de Educación, Departamento de Estudios y Desarrollo.

Hernández, R., Fernández, C. y Baptista, M. (2010). Metodología de la investigación. ( $5^{a}$ ed.). México D.F.: McGraw-Hill.

Marshall Infante, M. T. (2004). Programas de mejoramiento de las oportunidades. El Liceo para Todos en Chile. Santiago, Chile: UNESCO.

MINEDUC, Agencia de Calidad de la educación. (2012c). Plan de Evaluaciones Nacionales e Internacionales. Recuperado de http://www. agenciaeducacion.cl/wp-content/uploads/2013/02/Plan_de_ Evaluaciones_Actualizado.pdf. 
MINEDUC, Agencia de Calidad de la Educación. (2016). ¿Qué es el SIMCE? Recuperado de http://www.agenciaeducacion.cl/simce/que-es-elsimce/.

MINEDUC. (2008). Ley 20.248. División de Educación General. Recuperado de http://portales.mineduc.cl/usuarios/formacion_tecnica/File/20I I / NORMATIVA/Ley\%2020248\%20SEP.pdf.

MINEDUC. (2012a). Bases curriculares. Lenguaje y Comunicación. Santiago, Chile: Mineduc.

MINEDUC. (20I2b). Bases Curriculares. Matemática. Santiago, Chile: Mineduc.

MINEDUC. (2014). Orientaciones técnicas para sostenedores y directivos escolares. Plan de Mejoramiento Educativo. Nuevo enfoque 4 años. Recuperado de http://portales.mineduc.cl/usuarios/mineduc/File/20I4/PME\%20 DOC\%20NI\%20VF_20I4.pdf.

Pastrana, J., Fernández, M., Salinas, I., Gutierrez, P. y Nuñez, C. (2015). Desafíos de equidad en la educación chilena. Psicoperspectivas, I4(3), I-4.

Román, M. y Murillo, F. (2012). Políticas educativas de apoyo a escuelas de sectores pobres y de bajo logro académico en Chile: I990-20I I. Revista de Educación, 46-66.

Superintendencia de Educación. (2016). Manual de cuentas para la rendición de recursos 2015 de recursos públicos destinados a la educación. Recuperado de http://ptf. supereduc.cl/docs/v2manualdecuentas.pdf.

Weinstein, J., Fuenzalida, A. y Muñoz, G. (2010). La subvención preferencial: desde una difícil instalación hacia su institucionalización. ¿Fin de Ciclo? Cambio en la Gobernanza del Sistema Educativo, I6I-I82. 